Sonographi c anal ysi s of hyoi d bone movement dur ing swal I owi ng

\begin{tabular}{|l|l|}
\hline 著者 & $\begin{array}{l}\text { Yabunaka Koi chi, Ohue Mit sumi, Hashi not o } \\
\text { Tsut om, Kat suda Toshi zo, Yamanto o Kenyu, } \\
\text { Sanada Shi ger u }\end{array}$ \\
\hline $\begin{array}{l}\text { j our nal or } \\
\text { publ i cat i on ti t l e }\end{array}$ & I FMBE Proceedi ngs \\
\hline vol une & 22 I FMBE \\
\hline page r ange & $840-842$ \\
\hline year & $2008-01-01$ \\
\hline URL & ht t p: //hdl . handl e. net /2297/19912 \\
\hline
\end{tabular}




\title{
Sonographic analysis of hyoid bone movement during swallowing
}

\author{
Koichi Yabunaka ${ }^{1}$, Mutsumi Ohue ${ }^{2}$, Tsutomu Hashimoto ${ }^{3}$, \\ Toshizo Katsuda ${ }^{4}$, Kenyu Yamamoto ${ }^{5}$ and Shigeru Sanada ${ }^{6}$ \\ ${ }^{1}$ Department of Radiology, Katsuragi Hospital, Osaka, Japan \\ ${ }^{2}$ Department of Orthopedics, Katsuragi Hospital, Osaka, Japan \\ ${ }^{3}$ Department of Rehabilitation, Katsuragi Hospital, Osaka, Japan \\ ${ }^{4}$ Faculty of Health Care Sciences, Himeji Dokkyo University, Himeji, Japan \\ ${ }^{5}$ Graduate School of Health Sciences, Okayama University, Okayama, Japan \\ ${ }^{6}$ Graduate School of Health Sciences, Kanazawa University, Kanazawa, Japan
}

\begin{abstract}
Purpose: Videofluoroscopy is generally considered a "gold standard" technique; however this technique exposes subjects to radiation. Ultrasonography is applied widely in clinical practice because of low cost, safety of the technique, and absence of radiation exposure. The aim of this study was to evaluate hyoid bone movement trajectories during swallowing in healthy subjects using ultrasonography.Subject and Methods: Data were obtained from 15 healthy volunteers (mean age: $34.9 \pm 9.3$ ). The subjects were divided into 3 groups: 5 subjects in their 20s, 5 subjects in their 30s and 5 subjects in their 40s. The subjects were examined while sitting in an upright position, with the back against a wall to control movement. The transducer was placed in a longitudinal scan above the larynx. The hyoid bone was identified on the scan as a high echoic area with posterior acoustic shadow. Then, the subjects swallowed $5 \mathrm{~mL}$ of mineral water, with a measurement frequency of 5 times. The digital-ultrasonographic recordings were viewed at $30 \mathrm{frames} / \mathrm{s}$ for 3 seconds. Results: The movement was divided into 4 phases: A, slowly ascending phase; $B$, rapidly ascending phase; $\mathrm{C}$, temporary pause phase (position of maximum rise); $\mathrm{D}$, rapid and slow descending shifts toward the resting position phase. We easily visualized the hyoid bone trajectory using ultrasonography. In all cases, ultrasonographic analysis of the hyoid bone was confirmed to have a similar trajectory as determined with videofluoroscopy. Ultrasonography can accurately determine swallowing duration and trajectory of hyoid bone movement. There was a significant difference in swallowing duration between the 20s groups and 30s groups and between the 20 s groups and 40 s groups $(P<0.05)$. However, the difference between the 30s group and 40s group was not significant $(P=0.87)$.Conclusion: The results show that the dynamic phase image of the hyoid bone movement can be revealed by ultrasonographic visualization.
\end{abstract}

Keywords-Ultrasonography, Hyoid bone motion, Swallowing,

\section{I .INTRODUCTION}

Feeding and swallowing dysfunction is of greater concern now than in the past due to the increased numbers of elderly people, and the potential for increased risk of pneumonia from dysphagia with frequent aspiration. Videofluoroscopy is generally considered the "gold standard” method for diagnosing dysphagia [1-3]. However, this procedure has a number of limitations, including exposure of patients to radiation and the fact that it requires contrast medium. Transabdominal ultrasonography (US) is applied widely in clinical practice because of low cost, safety of the technique, speed, and absence of radiation exposure. Few studies have analyzed hyoid bone movement by US [4,5]. Additionally, swallowing has not been analyzed. The aim of this study was to evaluate hyoid bone movement trajectories during swallowing in healthy subjects, using ultrasonography..

\section{II .MATERIALS AND METHODS}

\section{A. Patients}

A total of 30 subjects underwent swallowing sonographic examination between December 2007 and March 2008 at Katsuragi Hospital (Osaka, Japan). Data were obtained from 15 healthy volunteers, and the subjects were divided into 3 groups: 5 subjects were in their 20s ( 2 men and 3 women; mean age, $24.2 \pm 2.3$ ), 5 were in their 30s (2 men and 3 women; mean age, 34.3 \pm 3.7) and 5 were in their 40s (3 men and 2 women; mean age, $45.4 \pm 2.1$ ). The study was approved by the ethics committee of our institution, and informed consent was obtained from all patients.

\section{B. Methods (Fig. 1)}

The subjects were examined sitting in the upright position, with their back resting against a wall to control movement. With the transducer placed in a longitudinal scan above the larynx, the hyoid bone was located in the middle of the screen. The measuring point to be located was the maximal 
position of the cranial hyoid bone, and the monitor displayed the cranial part on the left side and the caudal part on the right side. The hyoid bone was identified on the scan as a high echoic area with a posterior acoustic shadow. The subjects swallowed $5 \mathrm{~mL}$ of mineral water and measurements were taken 5 times. The moving images were preserved on a hard disk using ultrasonic equipment. Xario ultrasound systems were used with curved-array (3.5-7.0 $\mathrm{MHz}$ ) transducers (Toshiba Medical Systems, Tokyo, Japan).

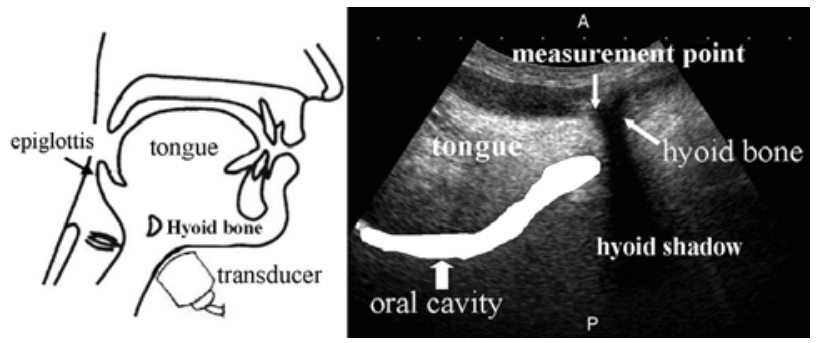

Fig1. A cross section of the head around the mouth, jaw and pharynx.

\section{Swallowing analysis (Fig. 2)}

The images were analyzed by software (image J), and digital-sonographic recordings were viewed at 30 frames/s for 3 seconds. Frozen frames of the moving image were analyzed, and range of movement of the hyoid bone from the resting point was measured ( $\mathrm{X}$ axis, horizontal migration length; $\mathrm{Y}$ axis, perpendicular migration length). We measured the time intervals between A and B, B and C, $\mathrm{C}$ and D. A corresponds to initiation of hyoid motion, B indicates full elevation and beginning of anterior motion, C corresponds to maximum anterior displacement and beginning of return motion and $\mathrm{D}$ indicates completion of the return to original resting position. The total swallowing duration was calculated by determining the interval from A to $\mathrm{D}$ in the three groups.
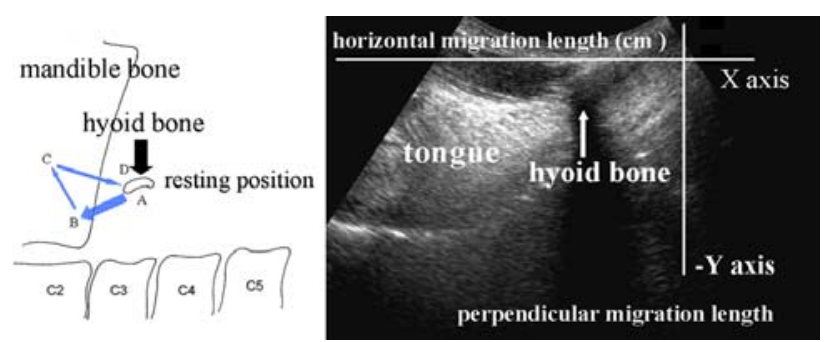

Fig2. Hyoid bone trajectory by videofluoroscopy. Sonograms show the range of hyoid bone movement from the resting point by coordinate graphics.

\section{Statistical analysis}

One-way analysis of variance was used to compare the mean swallowing duration times among the groups.
Statistical analyses were performed with SPSS 10.0 software, and $\mathrm{P}<0.05$ was considered significant.

\section{III.RESULTS}

\section{A. Analysis of hyoid bone movement}

In all cases, normal hyoid bone trajectory was easily visualized by ultrasonography. Figure 3 shows the trajectories of 5 swallows of one normal subject. This figure demonstrates that a normal swallow generally consists of four phases. First, the hyoid bone rises from its resting position (A-B, elevation) after swallowing was initiated. Second, the hyoid bone moved anteriorly to reach its maximum displacement (B-C, anterior). Third, the hyoid bone remained in a temporary phase (position of maximum rise). Finally, the hyoid bone returned to its resting position (C-D, return).

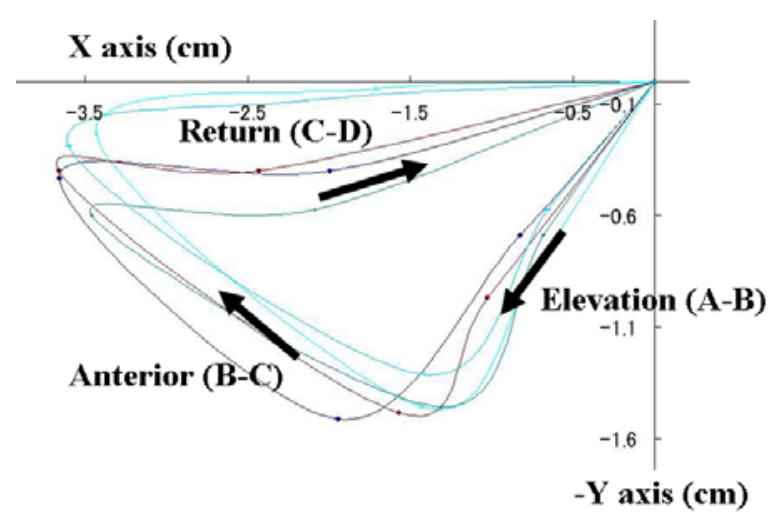

Fig3. Normal hyoid bone trajectory of 5 consecutive swallows (5-mL water boluses) in a 24-year-old female.

\section{B. Swallowing duration times in the three groups}

Table 1 shows the average duration measurements (Elevation, Anterior, Remain and Return) of 3 groups for 5 control swallows of $5 \mathrm{~mL}$ of water. Regarding the duration of measurements of 15 normal subjects, significant differences were found between the 20s group or 30s group and 40s group $(\mathrm{P}<0.05)$, but differences between the $30 \mathrm{~s}$ group and 40s group were not significant $(\mathrm{P}=0.87)$.

Table 1 Average duration measurements of three groups (S)

\begin{tabular}{|c|c|c|c|c|c|}
\hline & Total time & Elavation & Anterior & Remain & Return \\
\hline Twenties & $1.48 \pm 0.2$ & $0.41 \pm 0.1$ & $0.32 \pm 0.1$ & $0.21 \pm 0.1$ & $0.54 \pm 0.2$ \\
\hline Thirties & $1.81 \pm 0.2$ & $0.51 \pm 0.2$ & $0.45 \pm 0.1$ & $0.26 \pm 0.1$ & $0.59 \pm 0.2$ \\
\hline Forties & $1.87 \pm 0.2$ & $0.55 \pm 0.2$ & $0.38 \pm 0.1$ & $0.31 \pm 0.1$ & $0.64 \pm 0.3$ \\
\hline Total & $1.73 \pm 0.3$ & $0.49 \pm 0.2$ & $0.38 \pm 0.1$ & $0.26 \pm 0.1$ & $0.59 \pm 0.2$ \\
\hline
\end{tabular}

\section{IV.DisCUSSION}


In this study, the trajectory of the hyoid bone identified by ultrasonographic analysis was confirmed to be similar to that by videofluoroscopy. Thus, ultrasonography can accurately determine the swallowing duration and trajectory of hyoid bone movement. However, the hyoid bone trajectories depicted shift length of movement in the same subject. Some studies have reported that the position of the head influences pharyngeal swallowing [6,7]. One of the reasons for the different trajectory in this study may have been that the head was tilted (anteflexion or retroflexion of the neck) at a slight angle. Therefore, in the future, an examination technique will be necessary to obtain a stabilized, reproducible image of the fixed head. Based on the ultrasonographic observation, swallowing affected the jaw and cartilago thyroidea; therefore, a convex type or sector type probe should be used.

The average swallowing duration measurements increased with age. The reason might be that hyoid bone elevation is lowered due to atrophy of the surrounding muscles (digastric muscle, mylohyoid muscle, stylohyoid muscle, geniohyoid muscle), resulting in less tension and loosening of ligaments during swallowing. This study used drinking water for convenient samples; however, any test foods or drinks can be chosen. Thus, we will plan to examine the effect of food on hyoid bone motion.

This study had some limitations. We did not include subjects greater than 50 years old nor did we have subjects with swallowing disorders. Moreover, the number of subjects was small. Therefore, we plan to study hyoid bone movement in older subjects and those with dysphagia in the future.

\section{Conclusions}

The results show that dynamic phase images of the hyoid bone movement can be obtained using ultrasonographic visualization. Similar results can be obtained using US and $\mathrm{x}$-ray videofluoroscopy. Ultrasonography can be used for determining the position of the hyoid bone, which may prove useful for patients with dysphagia or other swallowing disorders.

\section{REFERENCES}

1. Logemann JA, Rauloski BR, Rademaker AW et al. (2000) Temporal and biomechanical characteristics of oropharyngeal swallow in younger and older men. J Speech Lang Hear Res 43: 1264-1274.

2. Logemann JA, Rauloski BR, Rademaker AW et al. (2002) Oropharyngeal swallow in younger and older women: videofluoroscopic analysis. J Speech Lang Hear Res 45: 434-445.

3. Chi-Fishman G. (2005) Quantitative lingual, pharyngeal and laryngeal ultrasonography in swallowing research: a technical review. Clin Linguist Phon 19: 589-604.

4. Sonies BC, Wang C, Sapper DJ. (1996) Evaluation of normal and abnormal hyoid bone movement during swallowing by use of ultrasound duplex-Doppler imaging. Ultrasound Med Biol 22: 11691175.

5. Kuhl V, Eicke BM, Dieterich M et al. (2003) Sonographic analysis of laryngeal elevation during swallowing. J Neurol 250: 333-337.

6. Logemann JA, Kahrilas PJ, Kobara M et al. (1989) The benefit of head rotation on pharyngoesophageal dysphagia. Arch Phys Med Rehabil 70: 767-771.

7. Ekberg O. (1986) Posture of the head and pharyngeal swallowing. Acta Radiol Diagn (Stockh) 27:691-696.

address of the corresponding author:

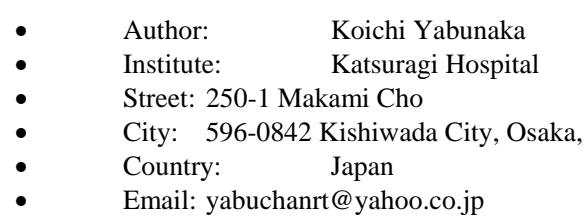


Table 1 Average duration measurements of three groups (S)

$\left.\begin{array}{lccccc}\hline & \text { Total time } & \text { Elavation (A-B) } & \text { Anterior (B-C) } & \text { Remain } & \text { Return (C-D) } \\ \hline \text { Twenties } & 1.48 \pm 0.22 \\ \text { Thirties } & 1.81 \pm 0.2\end{array}\right] * \begin{array}{r}0.41 \pm 0.1 \\ \text { Forties } \\ \text { Total }\end{array}$



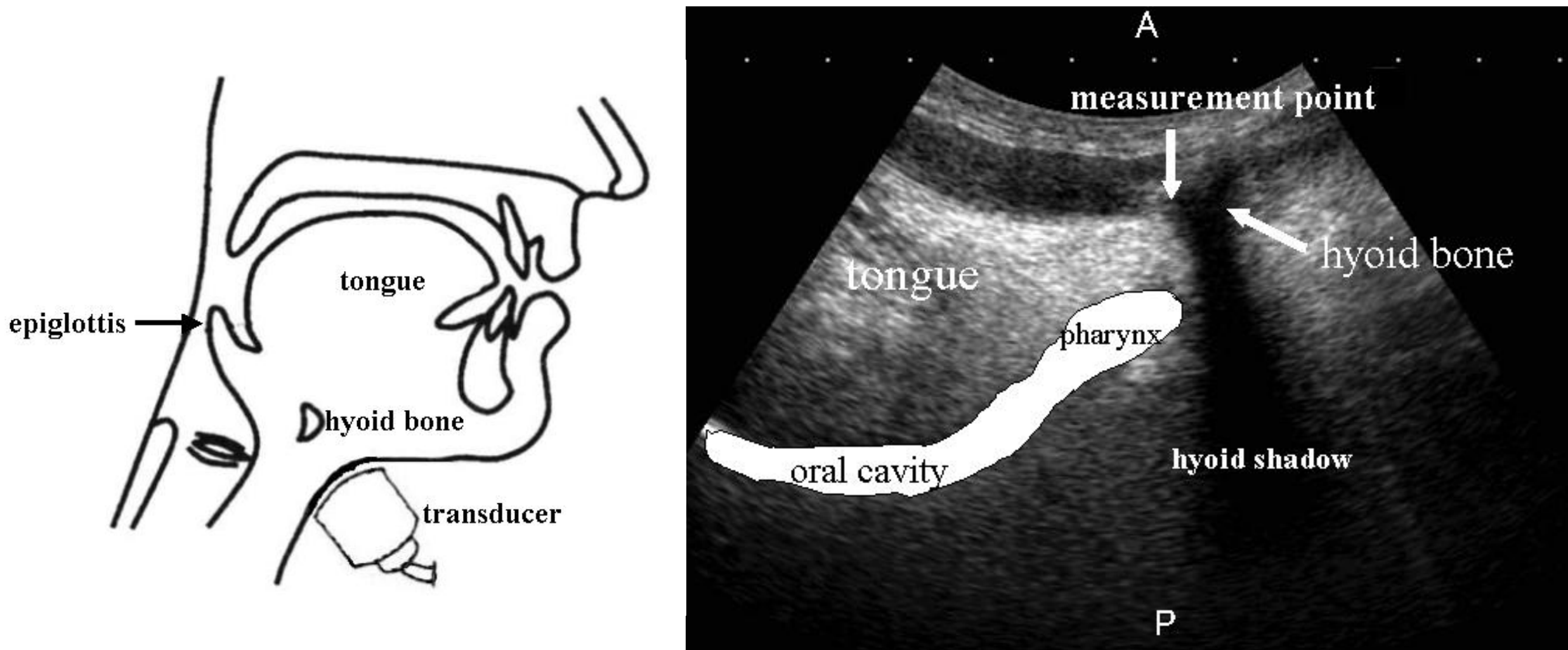

Fig 1. Diagram shows the submental position of the transducer and settnig of the curved-array transducers during scanning of the hyoid bone in the sagittal plane. Sonograms show the measuring point of hyoid bone. 

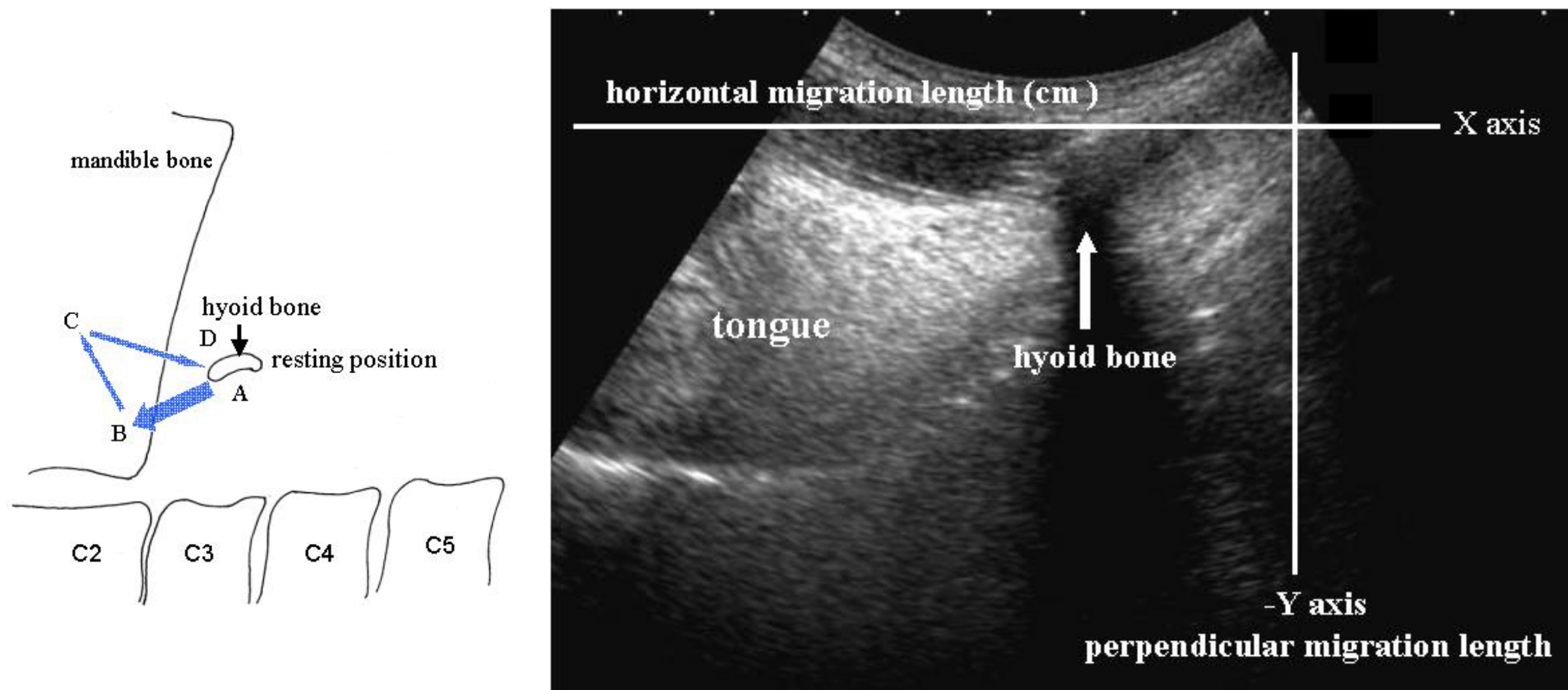

Fig 2. Diagram shows hyoid bone trajectory by videofluoroscopy. Sonograms show range of hyoid bone movement from the resting point by coordinate graphics. 


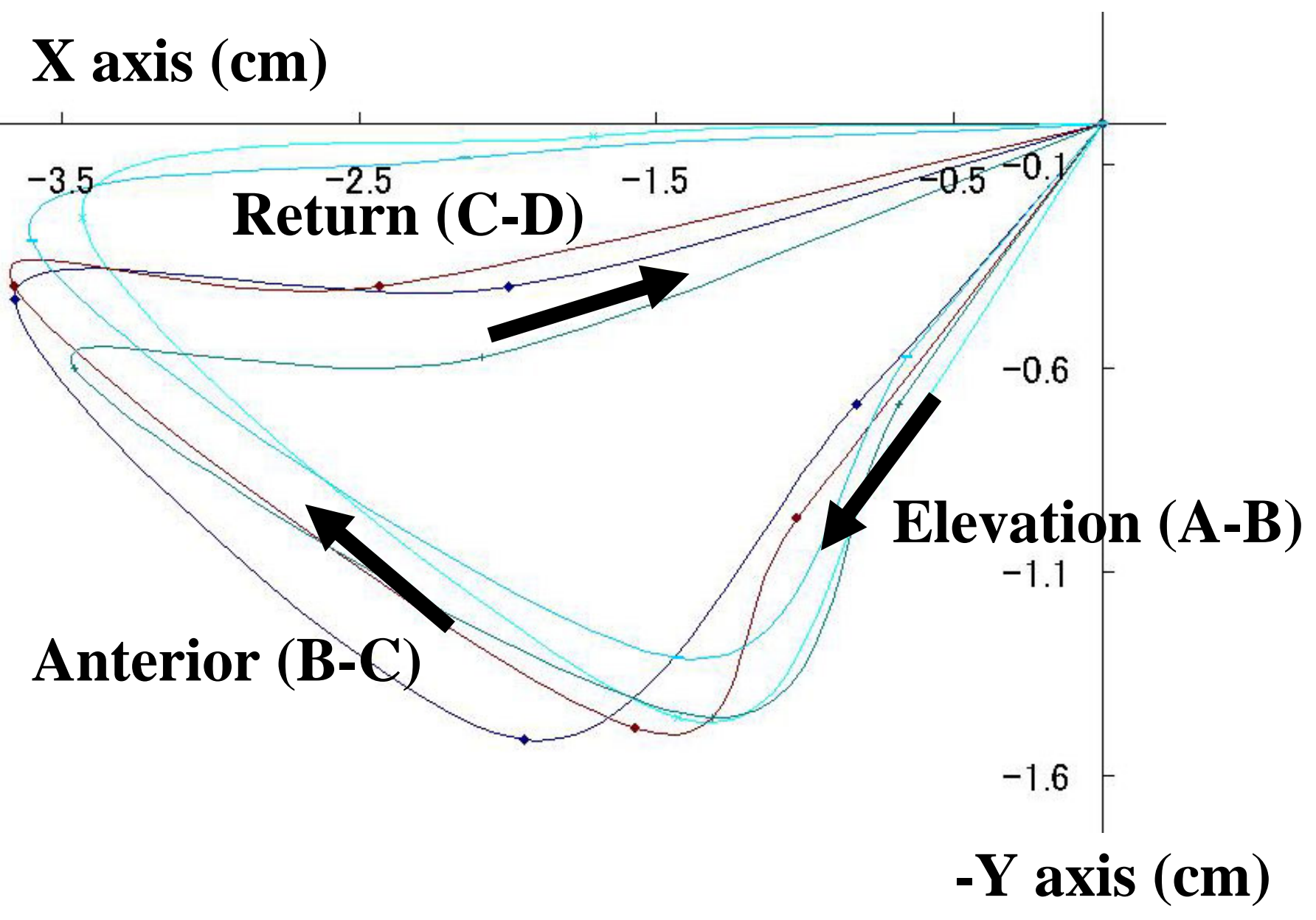

Fig 3. The normal hyoid bone trajectory of data of 5 consecutive swallows (5-ml water boluses) in a 24-year-old female. 